\title{
Stereotactic radiosurgery for vestibular schwannomas
}

\section{Steve Braunstein Lijun Ma}

Department of Radiation Oncology, University of California San Francisco, San Francisco, CA, USA
Correspondence: Steve Braunstein Department of Radiation Oncology, University of California San Francisco, 505 Parnassus Avenue, San Francisco, CA 94143, USA

Tel +l 4I5 3538900

Fax + I 4153538679

Email steve.braunstein@ucsf.edu
This article was published in the following Dove Press journal: Cancer Management and Research

\begin{abstract}
Stereotactic radiosurgery (SRS) maintains an important role in managing vestibular schwannoma (VS). Long-term clinical data have clearly established the safety and efficacy of the procedure for managing Koos low grade to intermediate grade VS. Historically, the procedure was developed via a multidisciplinary approach that involves physicians (eg, neurosurgeons and radiation oncologists) as well as clinical specialists (eg, radiation physicists). In this paper, we have reviewed current technical and clinical practices of SRS for VS from a procedural specialist's perspective and from a clinician's perspective.
\end{abstract}

Keywords: acoustic neuroma, vestibular schwannoma, radiosurgery, gamma knife

\section{Introduction}

The goal of this paper was to highlight the protocols and data that are relevant to the current clinical practice and technical standards for managing VS with stereotactic radiosurgery (SRS).

\section{Technical perspective State-of-the-art SRS modalities}

As first coined by Dr Lars Leksell, the term "stereotactic radiosurgery (SRS)" indicates direct application of a precise spatial localization apparatus for a procedure that delivers a high dose of radiation accurately to a lesion inside the brain. ${ }^{1}$ The original localization apparatus envisioned by Dr Lars Leksell entailed a fixation metal frame (ie, stereotactic frame) in conjunction with the use of orthovoltage X-rays. Subsequently, technological advancements quickly replaced low-energy $\mathrm{X}$-rays with megavoltage X-rays or high-energy gamma rays. Megavoltage X-rays are primarily produced from $\mathrm{C}$-arm gantry-mount medical linear accelerators, and gamma rays are primarily produced from high-activity radioactive sources such as ${ }^{60} \mathrm{Co}$, where its spectroscopy profile reveals two photon peaks at the energies of 1.17 and $1.33 \mathrm{MeV}$, respectively.

Besides high-energy gamma rays or X-rays, mechanical alignment accuracy is another hallmark of the SRS procedure, whereby all of the radiation beams are aligned precisely toward a focal point in space, namely the isocenter. Current state-of-the-art SRS systems typically maintain mechanical beam alignment accuracy of $0.5 \mathrm{~mm}$ or less. Such a high standard of accuracy was historically set with the early Leksell Gamma Knife system that was pioneered by Dr Lars Leksell in the 1960s. ${ }^{2}$ 
For example, the mechanical accuracy of the first Gamma Knife model U system installed in North America was reported to be less than $0.25 \mathrm{~mm} .{ }^{3}$ This unit was delivered to the University of Pittsburgh in 1987 and the whole system weighed more than 20 tons with nearly $6,000 \mathrm{Ci}$ of ${ }^{60} \mathrm{Co}$ sources loaded and placed around a hemispherical surface to form a $2 \pi$ solid angle. As a result, 201 individually shaped beams were individually aligned toward a single isocenter making the misalignment to $<0.002 \mathrm{~mm}$ per beam, a remarkable engineering achievement for the system. Consequently, the alignment accuracy of submillimeter isocenter has become the gold standard for benchmarking all SRS systems, especially applicable to the modern linac-based SRS systems.

Based on the number of isocenters typically used for treating VS, modern state-of-the-art SRS modalities can be classified into three types: 1) Leksell Gamma Knife system, including the recently released the Leksell Gamma Knife Icon system (Elekta AB, Stockholm, Sweden), where multiple isocenters (eg, $\mathrm{N}>3$ ) are typically employed for treating a VS lesion; 2) Robotic X-band linear accelerator such as the latest CyberKnife M6 model (Accuray, Sunnyvale, CA, USA), where non-isocenter beams on the order of a few hundreds are often used for treating VS; and 3) $\mathrm{C}$-arm-based S-band linear accelerator such as the TrueBeam STx or Edge Model (Varian Oncology System, Palto Alto, CA, USA), where a single isocenter with multiple fixed or rotational arc beams are often employed for SRS treatment of VS. All these systems assert sub-millimeter beam alignment and mechanical accuracy on the order of $0.5 \mathrm{~mm}$ or less. ${ }^{4}$

Such a high degree of beam alignment accuracy enables multiple cross-firing beams and/or multiple isocenters to be directed and superimposed inside an irregularly shaped target volume (such as the VS lesion). Cross-firing multiple beams from different angles toward a single or multiple isocenters are essential for SRS of VS in order to achieve a conformal dose distribution and adequate dose coverage of the target while sparing adjacent normal structures such as the cochlea and the brainstem.

Historically, stereotactic frame was used to establish the stereotactic coordinate system for the purpose of precisely aligning and focusing multiple beams for an SRS treatment. With the developments of linac flattening-filter-free (FFF) technology and in-room or on-board imaging guidance system such as stereoscopic $\mathrm{kV}$ imaging system of the CyberKnife system and on-board imaging system for the
S-band linear accelerator, frameless SRS was introduced as an alternative solution to the traditional frame-based treatment. ${ }^{5,6}$ Notably, the latest GK Icon system also incorporated an on-board imaging system to provide frameless SRS solution in addition to the traditional frame-based SRS solution. ${ }^{7,8}$

One of the main issues of the frameless SRS is the intrafractional target shifts during the treatment that often require continuous monitoring and frequent corrections of the patient setups. This is in contrast to the framebased SRS where intrafractional target shifts are assumed minimal due to rigid frame fixations. The use of highdose-rate FFF beams for frameless SRS in part alleviated the problem by enabling the treatment to be delivered in minutes. However, concern for negative dose impact from potential interfractional target shifts particularly during a short treatment time remains. As a result, frameless SRS of VS has been largely employed and reported for fractionated treatments while frame-based treatments are almost exclusively used for single-fraction SRS. Both framebased and frameless SRS of VS aim to leverage the highly conformal dose distributions created from the multi-beam cross-firing technique.

An example of VS case illustrating SRS beam crossfiring principle is shown in Figure 1. In this example, multiple isocenters $(\mathrm{N}>5)$ are applied and the stereotactic coordinates (ie, $x=116.8 \mathrm{~mm}, y=85.0 \mathrm{~mm}, \mathrm{z}=115.4 \mathrm{~mm}$ ) of the first isocentric beam delivery are indicated in the pop-up menu on the lower right corner. In addition, the cross-firing confocal beams aiming toward the first isocenter are purposely shaped from several beam directions (cf, the pie diagram in the menu). In this case, all the beams surrounding the target are divided into eight independent sectors with each sector possessing variable beam sizes and directions. For example, sector $1=16 \mathrm{~mm}$ of the beam diameter and it is directed from the patient's anterior direction; sector $5=16 \mathrm{~mm}$ of beam diameter and it is directed from the patient's posterior direction; sector 2 to sector 4 $=16 \mathrm{~mm}$ of the beam diameter and it is directed from the patient's left side; sector 6 and sector $8=8 \mathrm{~mm}$ of the beam diameter and it is directed from the patient's right side, and sector $7=0 \mathrm{~mm}$ of the beam diameter or it is completely blocked. It should be noted that the combinations of these confocal beams of variable diameters create a conformal dose distribution surrounding the irregularly shaped VS target. It also facilitates the sparing of the brainstem that is adjacent to the target volume. 


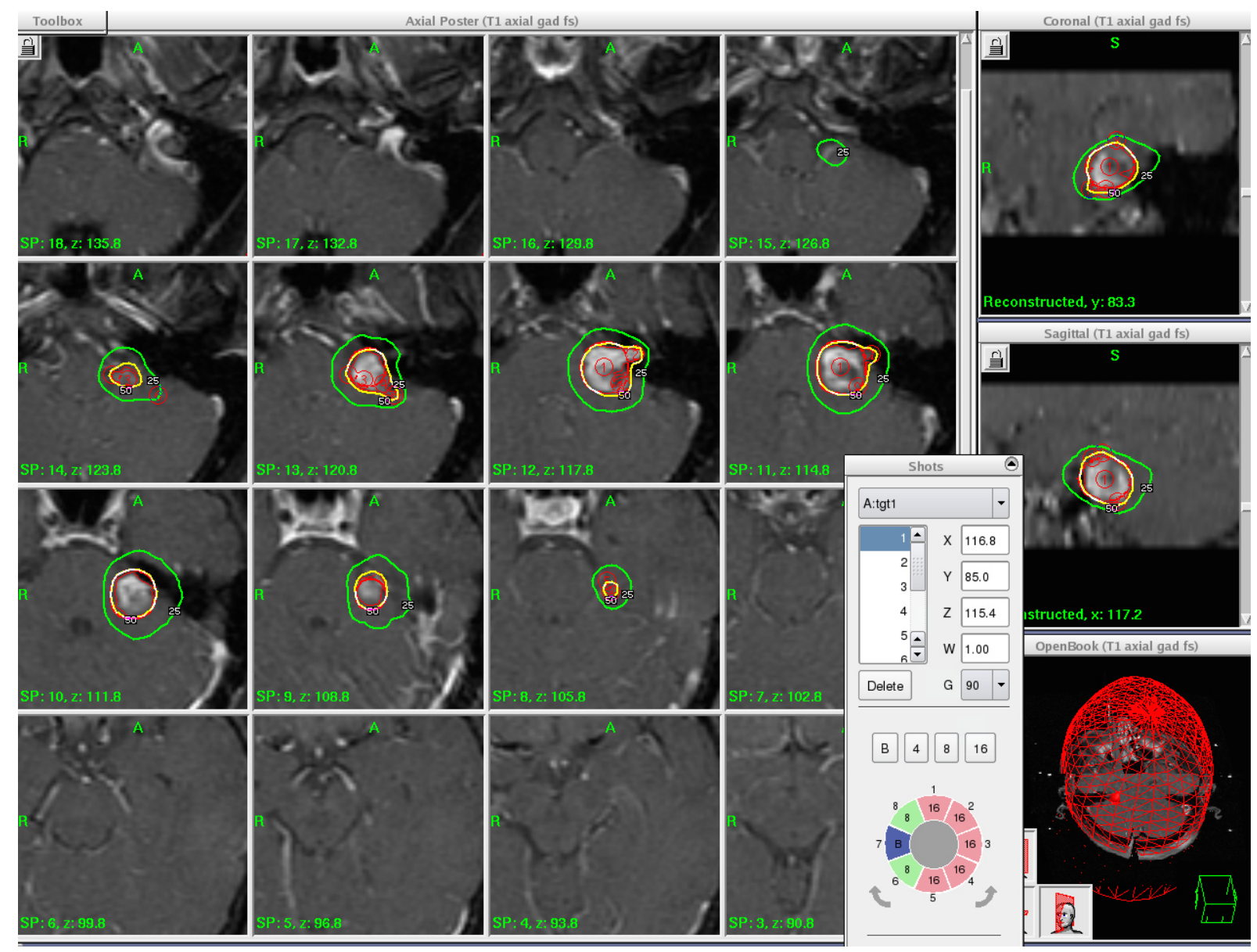

Figure I An illustration of multi-isocenter, multi-beam irradiation of a left-side VS lesion on a Gamma Knife Icon system, where utilization of multiple isocenters and multiple directional shaped beams of variable beam diameters create the desired dose distribution.

Abbreviation: VS, vestibular schwannoma.

\section{Treatment planning}

High-resolution MR imaging capability is critical for SRS of VS for the purpose of soft-tissue contrast. Volumetric thinslice MR imaging (such as 3D fast spoiled gradient-echo sequence with $1.0-1.5 \mathrm{~mm}$ in slice thickness) with gadolinium contrast is typically employed for contouring the tumor volume. Volumetric T2 weighted MR imaging is often acquired for optic pathway structure definition and for identification of the cranial nerves as well as the cochlea. In order to resolve the bone interface within the target volume, volumetric $\mathrm{CT}$ imaging is also acquired to allow visualization of the target boundary as well as to enable crosscheck of the stereotactic coordinate definitions with the stereotactic coordinates from the volumetric MR studies. With sub-millimeter beam alignment accuracy, SRS beam targeting uncertainties for the majority of VS treatments are considered to be minimal. As a rule of thumb, margins of less than $2 \mathrm{~mm}$ are generally employed when defining the planning target volume (PTV) based on the contrast enhancement volume of the gross target volume (GTV).

Furthermore, the historical data of SRS of VS were predominantly based on the clinical experiences of Gamma Knife radiosurgery (GKSRS), where the GTV to PTV margin was routinely set to $0 \mathrm{~mm}$. As a result, the term "target volume" was widely cited without causing an ambiguity as to whether it refers to GTV or PTV. This caveat is particularly important when defining and evaluating treatment planning indices for SRS.

In general, three indices are commonly adopted by the user or the treatment planning software to optimize and to analyze an SRS treatment plan quality: 1) selectivity index (SI), 2) Paddick conformity index (PCI), and 3) gradient index (GI). ${ }^{9,10}$ They are defined as follows:

$$
S I=\frac{T I V}{P I V(100 \%)}
$$




$$
\begin{gathered}
P C I=\frac{T I V^{2}}{T V^{*} P I V(100 \%)}=S I * \frac{T I V}{T V} \\
G I=\frac{P I V(50 \%)}{P I V(100 \%)}
\end{gathered}
$$

where TV is the target volume, PIV(100\%) is the isodose volume receiving $100 \%$ of the prescribed dose, $\operatorname{PIV}(50 \%)$ is the isodose volume receiving $50 \%$ of the prescribed dose, TIV is the target volume enclosed by the prescription isodose surface, that is, it equals to the union volume of the TV and $\operatorname{PIV}(100 \%)$.

It should be noted that all of the above indices are constructed based on the volume ratios of a selected isodose surface and the targets (either GTV or PTV). Physically, SI measures the target volume that coincides with the prescribed isodose volume. By definition, SI increases as TIV increases for a given PIV $(100 \%)$. It should be noted that SI $=1.0$ when the prescription isodose surface completely falls inside a target. In other words, SI is a parameter that detects over-coverage of the target volume by the prescribed dose. In comparison, PCI accounts for the target volume coverage by multiplying SI with the percentage of target volume coverage. Evidently SI $=$ PCI if $100 \%$ target volume coverage is achieved. Ideally, PCI $=1.0$ for a perfect dose coverage and dose conformity. In reality, PCI ranges between 0.5 and 0.9 for a VS treatment, and the higher the PCI value, the more conformal the SRS treatment plan. However, for cases where normal structure sparing plays an important role such as to avoid excess irradiation to the facial and cochlear nerves, PCI may be significantly lower due to intentional under coverage of the target volume (ie, significantly lower TIV/TV value in Equation 2).

Besides SI and PCI, GI measures the peripheral isodose falloff in the neighborhood of the target volume. From the expression of GI of Equation 3, by default, the lower the GI value, the sharper the dose fall off. A study examining the general dose falloff characteristics of various SRS lesion including VS treated with different SRS modalities has shown that a GI value of 2.83 would denote an average dose falloff following the classic inverse square law. If GI $>2.83$, it indicates shallower dose falloff versus the inverse square law and if GI $<2.83$, then it indicates a steeper dose falloff versus the inverse square law. For most single-fraction VS treatment cases, GI generally ranges between 2.6 and 3.1 depending on the target shape and complexity, accounting for the sparing of near-by critical structures such as the cochlea and the brainstem. For special large VS treatment with planned under coverage of the target plus frequent use of large collimators with more scattering, a wider range of GI values may result and the user should be cautious. This is discussed in the following section.

An example case illustrating SRS of VS is shown in Figure 2.

In Figure 2, a dose of 12.5 Gy was prescribed to the contrast-enhanced GTV. It should be noted that the target volume for the case was divided into two separate components near inferior part of the lesion (noted on the image slice at $\mathrm{z}=127.6$ ). This produced $\mathrm{SI}=0.64$ for the case. With $100 \%$ target coverage, $\mathrm{PCI}=0.64 * 1.0=0.64$. GI $=2.99$ suggests shallower dose falloff than the inverse square law.

\section{Normal structure dose limits}

Brainstem and cochlea are the major normal structures for SRS of VS. When SRS was first applied for treating VS, a peripheral dose as high as $18-20$ Gy was used. Due to observed toxicities, the prescription dose was subsequently reduced to $12-14$ Gy while still demonstrating equivalent local control. The latest ASTRO Quantec guideline also recommends an SRS dose of 12-14 Gy to preserve hearing. Given such a prescription dose to the target, the tolerance dose of the brainstem as specified by the AAPM 101 report (eg, the point maximum dose of $15 \mathrm{~Gy}$ and no more than 0.5 cc receiving a dose of $10 \mathrm{~Gy}$ ) is readily satisfied for majority of VS cases treated with single fraction SRS.

On the other hand, due to the proximity of cochlea (often for $<1 \mathrm{~mm}$ from the target periphery), minimizing the dose to the cochlea is significantly more challenging than sparing the brainstem. Figure 3 illustrated such a case where the target and the cochlea were visualized on a T2 MR imaging study.

In the case of Figure 3, a tumor periphery dose of 12.5 Gy was prescribed and significant beam shaping as illustrated in Figure 1 was applied. As a result, the cochlea received a mean dose of $4.5 \mathrm{~Gy}$. As noted from Figure 3, the contoured structure of the cochlea is relatively small (eg, $<0.1 \mathrm{~mL}$ ). Various dose parameters besides the mean dose have been reported for the purpose of correlating a dose-response for SRS of VS.

Three most common dose surrogates for cochlea were 1) the point maximum dose, 2) central modiolus dose, and 3) volume-average mean dose. All of these have been reported as useful parameters to correlate with the hearing outcome post SRS. ${ }^{11-13}$ A study reported an inherent functional relationship 

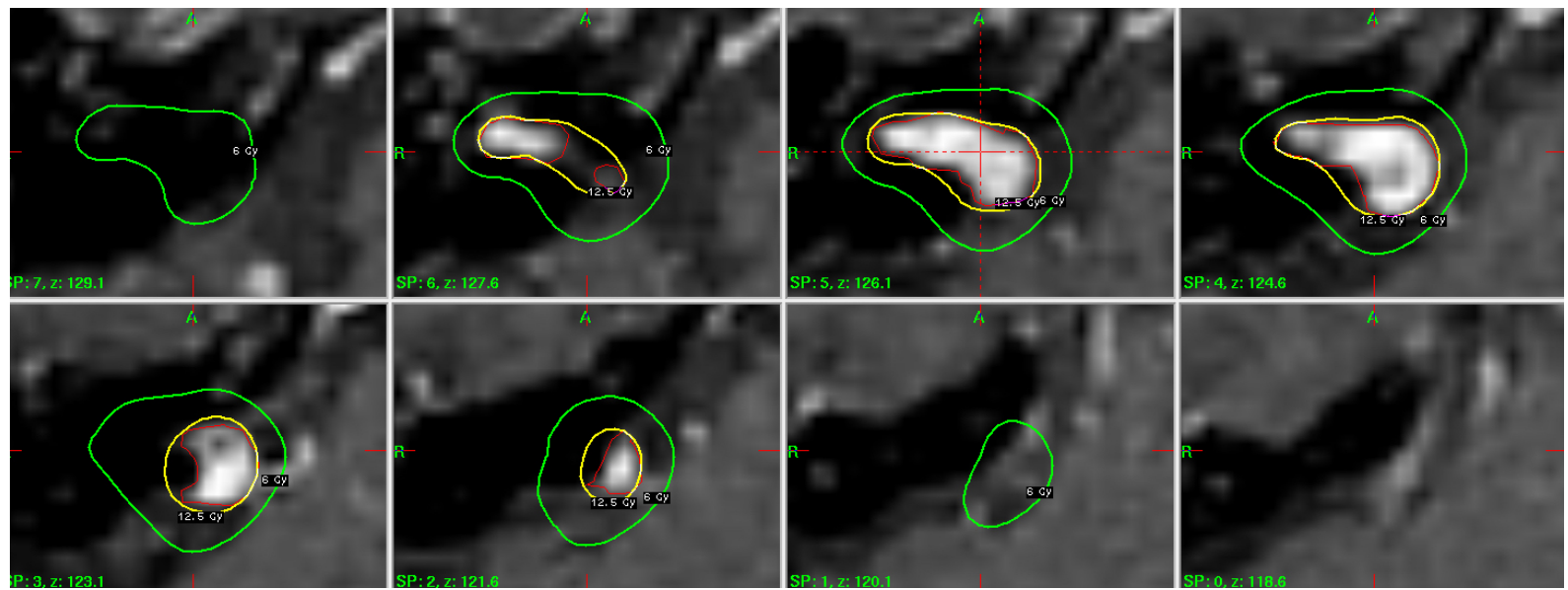

Figure 2 Axial dose distribution on a VS target volume superimposed onto the TI post-contrast serial MR scans with a slice thickness of I.5 mm. Abbreviation: VS, vestibular schwannoma.
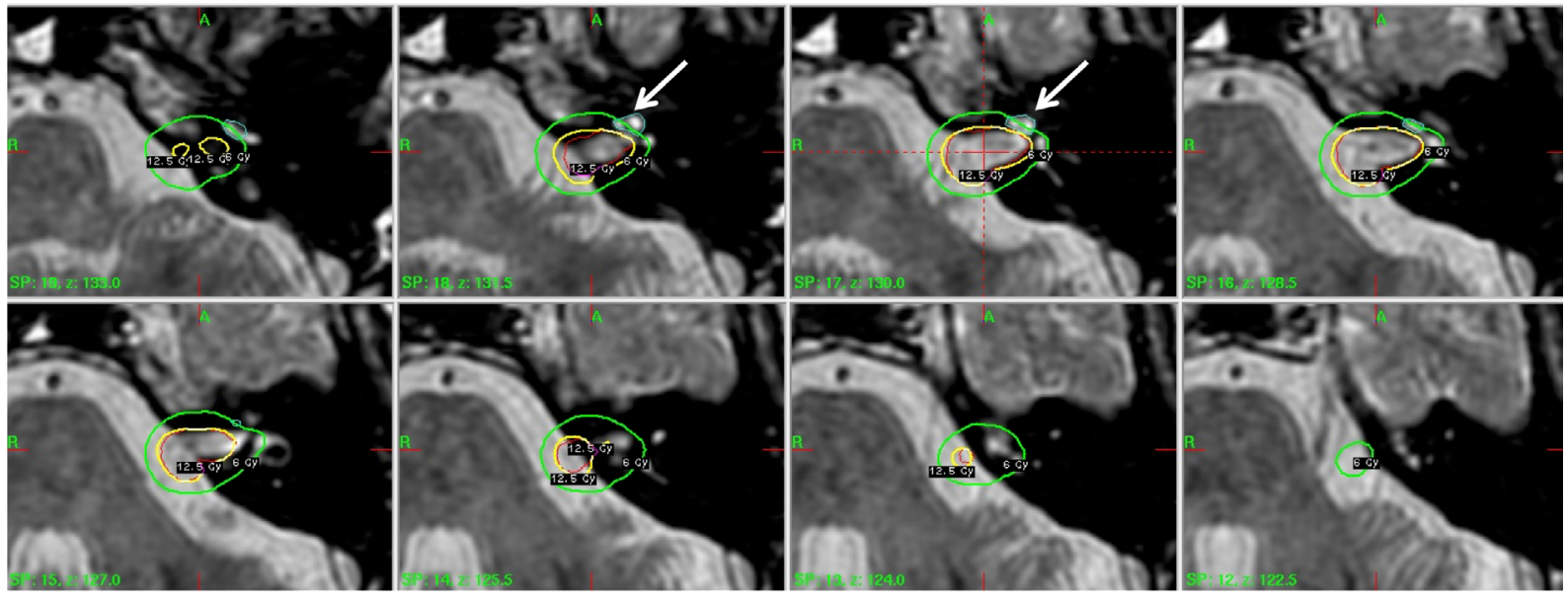

Figure 3 Illustration of dose distribution for SRS of a left-side VS case with the goal of minimizing the dose to the cochlea whose location is indicated by the arrow. Abbreviations: SRS, stereotactic radiosurgery; VS, vestibular schwannoma.

among these dose surrogates and found significant variability among these dose parameters. ${ }^{14}$ All dose parameters were found to correlate with the hearing change for a cohort of patients who underwent SRS of VS.

In particular, the point maximum dose has been found to be most useful in differentiating the risk probabilities. With 95\% confidence level (CL), a table of equivalent cochlear dose surrogates was established among the point maximum cochlear dose, modiolus cochlear dose, mean cochlear dose, and the dose to small hot spot volumes (such as $0.01-0.3$ cc) (Table 1).

As shown in Table 1, a point maximum cochlear dose of $12 \mathrm{~Gy}$ is therefore equivalent to a mean cochlear dose of $5.6 \pm 0.1 \mathrm{~Gy}$, a modiolus cochlear dose of $6.0 \pm 0.2 \mathrm{~Gy}$, and so on. It is worth noting that the risk probabilities of sensory neuronal hearing loss (SNHL) at a given dose level such as the maximum dose of 12 Gy (ie, a mean dose of 5.6 Gy or a modiolus dose of 6.0) remain unknown. Current data are on the dose-response are limited and also conflictive when reporting the risk of SNHL at one dose level versus another. Nonetheless, a single fraction prescription dose of 12-14 Gy is a good general practice in minimizing the risk of SNHL. This corresponds to maintaining the point maximum cochlear dose to the level of 12-14 Gy or less.

\section{Clinical perspective}

VS is also known as the acoustic neuroma (AN) in the literature. Specifically, VS or AN arises from the Schwann cell of myelin sheath of the eighth cranial nerve. It is a benign lesion, typically with a slow growth rate of $1 \mathrm{~mm}$ or less per year. Most of VS occurred sporadically except for NF2 patients, where they tend to have significantly higher $(3-4 \times)$ incidence rate and bilateral lesions also occur more commonly in NF2 patients. The rate of incidence for sporadic VS also increases 
Table I Equivalent cochlear dose parameters from an inherent functional formula

\begin{tabular}{llllll}
\hline $\begin{array}{l}\text { Point maximum dose } \\
\text { (Gy) }\end{array}$ & $\begin{array}{l}\mathbf{D}(\mathbf{0 . 0 1} \mathbf{~ m L}) \\
\mathbf{G y})\end{array}$ & $\begin{array}{l}\mathbf{D}(\mathbf{0 . 0 2} \mathbf{~ m L}) \\
\mathbf{( G y )}\end{array}$ & $\begin{array}{l}\mathbf{D}(\mathbf{0 . 0 3} \mathbf{~ m L}) \\
\mathbf{( G y})\end{array}$ & $\begin{array}{l}\text { Mean dose } \\
\text { (Gy) }\end{array}$ & $\begin{array}{l}\text { Modiolus dose } \\
\text { (Gy) }\end{array}$ \\
\hline 10.0 & $6.7 \pm 0.1$ & $5.9 \pm 0.1$ & $5.2 \pm 0.1$ & $4.9 \pm 0.1$ & $5.2 \pm 0.2$ \\
12.0 & $7.7 \pm 0.1$ & $6.7 \pm 0.1$ & $6.0 \pm 0.2$ & $5.6 \pm 0.1$ & $6.0 \pm 0.2$ \\
14.0 & $8.6 \pm 0.2$ & $7.5 \pm 0.2$ & $6.7 \pm 0.2$ & $6.2 \pm 0.1$ & $6.7 \pm 0.2$ \\
15.0 & $9.0 \pm 0.2$ & $7.8 \pm 0.2$ & $7.0 \pm 0.2$ & $6.6 \pm 0.2$ & $7.0 \pm 0.2$ \\
\hline
\end{tabular}

Notes: $\mathrm{D}(0.01 \mathrm{~mL}), \mathrm{D}(0.02 \mathrm{~mL})$, and $\mathrm{D}(0.03 \mathrm{~mL})$ denote the doses to the isodose volumes of $0.01,0.02$, and $0.03 \mathrm{~mL}$, respectively. The error bars in the table indicate mean $\pm 2 \mathrm{SD}$.

with the age and generally peaks for patients of $40-50$ years of age. In general, lesion control for lesion in the setting of NF2 is inferior to those occurring sporadically. ${ }^{15}$

\section{Tumor grade}

Patients with VS are usually diagnosed with hearing loss and/or loss of speech discrimination from the pure tone and speech audiogram. The tumor is generally classified via the Koos grade, where Koos Grade 1 tumors are localized only within the internal auditory canal (IAC); Koos Grade 2 tumors affect the IAC and the cerebellopintine angle; Koos Grade 3 tumors encroach the brainstem; Koos Grade 4 tumors cause distortions of the fourth ventricle.

Besides the tumor size, modern reports have also shown that cystic lesions generally respond better to SRS versus noncystic lesions. In general, macrocystic lesions tend to respond the best compared to the non-cystic or microcystic lesions. ${ }^{16}$

\section{Treatment options and tumor control}

SRS of VS is mostly applied to Koos Grade 1 and Grade 2 tumors due to the delayed radiation response and concern for treatment-related toxicities with large target volumes. With a target peripheral dose of 12-14 Gy for a single fraction, the tumor local control is reported to exceed $90 \%$ for Grade 1 and Grade 2 tumors regardless of the treatment modalities, including GKSRS and linac-based SRS. ${ }^{17}$ For large tumors such as Koos Grade 4, micro-surgery is recommended for fast relief of the mass effect and to prevent additional tumor growth that is found to be typically more significant for large tumors compared to small tumors. It should be noted that SRS can be a viable option for treating residual or recurrent VS post microsurgery with a high tumor control rate of $90 \%$ and low incidence of complications. ${ }^{18}$

For small VS tumors, observation was proposed as a reasonable alternative for treatment management. However, several studies comparing SRS and observation have noted a detectable tumor growth rate of $\sim 0.7 \mathrm{~mm}$ per year. Once the tumor growth has been established from the serial imaging studies of a patient, SRS is considered as a better option over observation for treatment management. Moreover, hearing preservation outcomes are superior to early treatment of smaller lesions. ${ }^{19}$

Koos high-grade tumors tend to have worse outcome compared to the lower grade tumors. One study has shown that the 5-year progression-free survival can decrease by as much as $5 \%$ when the tumor volume increases by $\sim 3 \mathrm{cc}^{20}$ How to improve local control for large VS lesions remains a challenge for SRS of VS tumors.

\section{Patient follow-up and functional outcomes}

After the SRS procedure, patients typically receive followup MR scans every 6 months plus audiology and neurologic examinations. Based on the latest clinical data, hearing preservation post SRS reaches $\sim 70 \%$ after 5 years. ${ }^{21,22}$ Studies have indicated that hearing preservation tends to correlate with early treatments within the first 2 years of diagnosis and the patient's initial status such as the pure-tune average difference $<10 \mathrm{~dB}$ between both ears. ${ }^{19,21,22}$

Risks of neurological deficits following SRS are low with an estimated risk of facial neuropathy and trigeminal neuropathy of $\sim 1 \%-3 \% .{ }^{23}$ However, all patients should be aware of the risk of malignant transformation of VS post SRS, which has been reported at $0.01 \%-0.1 \%{ }^{24,25}$ Similarly, the risk of secondary malignancy remains exceedingly low at $2.4 \%$ at 15 years. ${ }^{26}$

Pseudo-progression of VS is also found post an SRS procedure, which means that some tumors tend to enlarge in a transient period of the first 1-3 years during the follow-up. ${ }^{27}$ For asymptomatic patients, observation is sufficient and for some patients close follow-ups may be needed to differentiate pseudo-progression from real significant progression within the first 3 years of completing the SRS procedures. Of note, in the case of true regrowth, repeat radiosurgery may be a safe and effective strategy. ${ }^{28}$

\section{Controversies and developments}

Single fractional SRS has established an excellent local tumor control rate in the range of $90 \%$. It is minimally invasive and 
the procedure is convenient for patient as the same-day procedure. However, the technical complexity of the procedure is high, and not all patients have an easy access to a dedicated SRS program. In contrast, conventionally fractionated radiotherapy of delivering 1.8-2 Gy fraction for 4-5 weeks has also shown to be effective for managing VS tumors. ${ }^{29}$

Hypofractionated SRS treatments with a removable stereotactic frame have also been explored for the purpose of further improving the local control and hearing preservations. For hypofractionated SRS, a GTV to PTV margin such as 2 $\mathrm{mm}$ is often included to account for intrafractional targeting uncertainties. It remains controversial as to the technique as well as to the dose fractionation schemes that would offer the best local dose control and/or the lower toxicity profiles versus the single fraction SRS..$^{30}$

Although single fraction SRS has shown to be highly effective for small VS, managing large VS with SRS remains controversial. ${ }^{31}$ Some investigators have proposed hypofractionated treatments or multi-session volume-staged approach of managing these challenging cases with SRS. In the case of volume-staging, a single fraction SRS is first applied to a partial tumor volume distal to critical structures with the expectation of tumor shrinkage. Once tumor shrinkage is confirmed on interval imaging, an additional SRS procedure would be performed to treat the residual target volume. Others have proposed a hybrid approach of planned subtotal resection (STR) followed by radiosurgery with excellent rates of hearing and facial nerve preservation. ${ }^{32}$

Some investigators have argued that the key surgical objective for managing large VS has been shifted over the last decade from maximum tumor removal to nerve preservation. In a recent meta-analysis of planned STR followed by SRS, such an approach has been shown to produce excellent functional outcomes with facial nerve preservation rate exceeded $95 \%$ and serviceable hearing preservation approaching $60 \%$ while achieving a tumor control rate of $94 \%{ }^{33}$ This is a significant result considering relative high morbidity that associated with the attempt of achieving total surgical resection of the tumor. ${ }^{34,35}$

From a technical perspective, further enhancing the dose falloff or "sharpening the edge" between the target and the normal structure remains to be a challenge for the next generation of SRS device. With the rapid advancements of online stereotactic imaging localization such as that realized in the latest Gamma Knife Icon system plus significant elevation of radiation beam output such as that realized in the modern digitally controlled FFF linear accelerators, the use of SRS for VS is expected to expand with improved quality and efficiency of treatment planning. Ongoing technical developments continue to make the treatment device more integrated in terms of on-the-fly imaging and fast beam deliveries. This will continue to make SRS treatment become more accessible to all VS patients.

\section{Summary}

In this paper, we reviewed major technical and clinical perspectives of SRS of VS. The reader should be aware that no large randomized trials are available to guide a user on the best clinical and technical practices for SRS of VS given the pioneering effort of GKSRS. Nonetheless, a plethora of retrospective studies has been performed by the early adopters of the GKSRS and the data continued to expand with the advancement of SRS technology. Furthermore, expert consensus practice guidelines from recently published international society of stereotactic radiosurgery are useful for a user to review SRS of VS.

In summary, SRS has played an important role in managing VS. It is our expectation that such a role will continue to dominate and expand with continued advancements in the SRS technologies.

\section{Disclosure}

The authors report no conflicts of interest in this work.

\section{References}

1. Leksell L. The stereotaxic method and radiosurgery of the brain. Acta Chir Scand. 1951;102(4):316-319.

2. Régis J, Tamura M, Guillot C, et al. Radiosurgery with the world's first fully robotized Leksell Gamma Knife PerfeXion in clinical use: a 200-patient prospective, randomized, controlled comparison with the Gamma Knife 4C. Neurosurgery. 2009;64(2):346-355; discussion 355-356.

3. Wu A, Lindner G, Maitz AH, et al. Physics of gamma knife approach on convergent beams in stereotactic radiosurgery. Int J Radiat Oncol Biol Phys. 1990;18(4):941-949.

4. Phillips MH, Stelzer KJ, Griffin TW, Mayberg MR, Winn HR. Stereotactic radiosurgery: a review and comparison of methods. J Clin Oncol. 1994;12(5):1085-1099.

5. Sahgal A, Ma L, Chang E, et al. Advances in technology for intracranial stereotactic radiosurgery. Technol Cancer Res Treat. 2009;8(4): 271-280.

6. Ma L, Wang L, Tseng CL, Sahgal A. Emerging technologies in stereotactic body radiotherapy. Chin Clin Oncol. 2017;6(Supp1 2):S12.

7. Aldahlawi I, Prasad D, Podgorsak MB. Evaluation of stability of stereotactic space defined by cone-beam CT for the Leksell Gamma Knife Icon. J Appl Clin Med Phys. 2017;18(3):67-72.

8. Tuleasca C, Leroy HA, Régis J, Levivier M. Gamma knife radiosurgery for cervical spine lesions: expanding the indications in the new era of Icon. Acta Neurochir. 2016;158(11):2235-2236.

9. Paddick I. A simple scoring ratio to index the conformity of radiosurgical treatment plans. Technical note. J Neurosurg. 2000;93(Suppl 3): 219-222.

10. Paddick I, Lippitz B. A simple dose gradient measurement tool to complement the conformity index. J Neurosurg. 2006;105(Suppl):194-201. 
11. Kano H, Kondziolka D, Khan A, Flickinger JC, Lunsford LD. Predictors of hearing preservation after stereotactic radiosurgery for acoustic neuroma. J Neurosurg. 2009;111(4):863-873.

12. Brown M, Ruckenstein M, Bigelow D, et al. Predictors of hearing loss after gamma knife radiosurgery for vestibular schwannomas: age, cochlear dose, and tumor coverage. Neurosurgery. 2011;69(3):605-613; discussion 613-614.

13. Paek SH, Chung HT, Jeong SS, et al. Hearing preservation after gamma knife stereotactic radiosurgery of vestibular schwannoma. Cancer. 2005;104(3):580-590.

14. Ma L, Braunstein SE, Theodosopoulos PV, Mcdermott MW, Sneed PK. Inherent functional dependence among cochlear dose surrogates for stereotactic radiosurgery of vestibular schwannomas. Pract Radiat Oncol. 2017;7(1):e1-e7.

15. Mathieu D, Kondziolka D, Flickinger JC, et al. Stereotactic radiosurgery for vestibular schwannomas in patients with neurofibromatosis type 2: an analysis of tumor control, complications, and hearing preservation rates. Neurosurgery. 2007;60(3):460-468; discussion $468-470$.

16. Bowden G, Cavaleri J, Monaco E, Niranjan A, Flickinger J, Lunsford LD. Cystic vestibular schwannomas respond best to radiosurgery. Neurosurgery. 2017;81(3):490-497.

17. Flickinger JC, Kondziolka D, Niranjan A, Maitz A, Voynov G, Lunsford LD. Acoustic neuroma radiosurgery with marginal tumor doses of 12 to 13 Gy. Int J Radiat Oncol Biol Phys. 2004;60(1):225-230.

18. Bailo M, Boari N, Gagliardi F, et al. Gamma knife radiosurgery for residual and recurrent vestibular schwannomas after previous surgery: clinical results in a series of 90 patients and review of the literature. World Neurosurg. 2017;98:60-72.

19. Kondziolka D, Mousavi SH, Kano H, Flickinger JC, Lunsford LD. The newly diagnosed vestibular schwannoma: radiosurgery, resection, or observation? Neurosurg Focus. 2012;33(3):E8.

20. Régis J, Pellet W, Delsanti C, et al. Functional outcome after gamma knife surgery or microsurgery for vestibular schwannomas. J Neurosurg. 2013;119(Suppl):1091-1100.

21. Régis J, Carron R, Delsanti C, et al. Radiosurgery for vestibular schwannomas. Neurosurg Clin N Am. 2013;24(4):521-530.

22. Baschnagel AM, Chen PY, Bojrab D, et al. Hearing preservation in patients with vestibular schwannoma treated with gamma knife surgery. J Neurosurg. 2013;118(3):571-578.
23. Kondziolka D, Lunsford LD, Mclaughlin MR, Flickinger JC. Long-term outcomes after radiosurgery for acoustic neuromas. $N \mathrm{Engl} \mathrm{J} \mathrm{Med.}$ 1998;339(20):1426-1433

24. Seferis C, Torrens M, Paraskevopoulou C, Psichidis G. Malignant transformation in vestibular schwannoma: report of a single case, literature search, and debate. J Neurosurg. 2014;121(Suppl):160-166.

25. Shin M, Ueki K, Kurita H, Kirino T. Malignant transformation of a vestibular schwannoma after gamma knife radiosurgery. Lancet. 2002;360(9329):309-310.

26. Pollock BE, Link MJ, Stafford SL, Parney IF, Garces YI, Foote RL. The risk of radiation-induced tumors or malignant transformation after single-fraction intracranial radiosurgery: results based on a 25-year experience. Int J Radiat Oncol Biol Phys. 2017;97(5):919-923.

27. Hayhurst $\mathrm{C}, \mathrm{Zadeh} \mathrm{G}$. Tumor pseudoprogression following radiosurgery for vestibular schwannoma. Neuro Oncol. 2012;14(1):87-92.

28. Yomo S, Arkha Y, Delsanti C, Roche PH, Thomassin JM, Régis J. Repeat gamma knife surgery for regrowth of vestibular schwannomas. Neurosurgery. 2009;64(1):48-54; discussion 54-55.

29. Champ CE, Shen X, Shi W, et al. Reduced-dose fractionated stereotactic radiotherapy for acoustic neuromas: maintenance of tumor control with improved hearing preservation. Neurosurgery. 2013;73(3):489-496.

30. Tsao MN, Sahgal A, Xu W, et al. Stereotactic radiosurgery for vestibular schwannoma: International Stereotactic Radiosurgery Society (ISRS) Practice Guideline. J Radiosurg SBRT. 2017;5(1):5-24.

31. Huang CW, Tu HT, Chuang CY, et al. Gamma knife radiosurgery for large vestibular schwannomas greater than $3 \mathrm{~cm}$ in diameter. $J$ Neurosurg. 2018;128(5):1-8.

32. Radwan H, Eisenberg MB, Sandberg Knisely JP, Ghaly MM, Schulder M. Outcomes in patients with vestibular schwannoma after subtotal resection and adjuvant radiosurgery. Stereotact Funct Neurosurg. 2016;94(4):216-224.

33. Starnoni D, Daniel RT, Tuleasca C, George M, Levivier M, Messerer M. Systematic review and meta-analysis of the technique of subtotal resection and stereotactic radiosurgery for large vestibular schwannomas: a "nerve-centered" approach. Neurosurg Focus. 2018;44(3):E4.

34. Samii M, Gerganov VM, Samii A. Functional outcome after complete surgical removal of giant vestibular schwannomas. J Neurosurg. 2010;112(4):860-867.

35. Silva J, Cerejo A, Duarte F, Silveira F, Vaz R. Surgical removal of giant acoustic neuromas. World Neurosurg. 2012;77(5-6):731-735.
Cancer Management and Research

\section{Publish your work in this journal}

Cancer Management and Research is an international, peer-reviewed open access journal focusing on cancer research and the optimal use of preventative and integrated treatment interventions to achieve improved outcomes, enhanced survival and quality of life for the cancer patient. The manuscript management system is completely online and includes

\section{Dovepress}

a very quick and fair peer-review system, which is all easy to use. Visit $\mathrm{http}: / /$ www.dovepress.com/testimonials.php to read real quotes from published authors. 